\title{
EXAMINATION OF AROMA PRODUCTION KINETICS OF DIFFERENT COMMERCIAL WINE YEASTS IN FERMENTING MUSCAT OTTONEL WINES WITH THE HELP OF SPME HEAD-SPACE SAMPLING AND FAST GC ANALYSIS
}

\author{
GY. VAS ${ }^{\mathrm{a}}$, I. BLECHSCHMIDT ${ }^{\mathrm{b}}$, T. KOVÁCS $^{\mathrm{c}}$ and K. VÉKEY ${ }^{\mathrm{d}}$ \\ a Research Institute for Viticulture \& Enology of Agricultural Ministry, H-3301 Eger, Kölyuktető \\ P.O. Box 83. Hungary; e-mail address: h12232vas@ella.hu \\ ${ }^{b}$ Frederich Schiller University, Faculty of Chemistry, Institute for Analytical and Inorganic Chemistry, \\ D-07745 Jena, Lessingstrasse 8. Germany; e-mail address: cbi@dagobert.rz.uni-jena.de \\ ${ }^{c}$ University of Horticulture and Food Industry, Department of Food Chemistry and Nutrition, \\ H-11 18 Budapest, Somlói út 14-16. Hungary \\ d Research Institute for Chemistry of the Hungarian Academy of Sciences, Laboratory of Mass \\ Spectrometry, H-1025 Budapest, Pusztaszeri u. 59-67. Hungary; e-mail address: vekey@cric.chemres.hu
}

(Received: 15 April 1998; accepted: 11 November 1998)

Solid phase microextraction (SPME) coupled to fast capillary gas chromatography was used for monitoring the wine fermentation process. This combination offers a simple, quick and sensitive approach suitable for characterization of head-space components of wines during the fermentation process without a complicated sample preparation procedure. In this work this method was used to observe the differences in aroma production between three different commercial yeasts and the indigenous yeast flora.

Keywords: fermentation, head-space sampling, fast GC analysis, SPME, wine yeast

Yeasts have a great effect on wine quality. Different yeasts liberate the glucosidally linked primary aroma compounds to different extent and synthesize the secondary aroma substances in various quantities. Thus, certain yeast strains are more suitable for the fermentation of the different grape musts than the others, because they make the aroma character of the cultivated grape varieties dominant in several different ways. The effect of yeasts is very important in winemaking. DiTTRICH (1992) in a Wachau lecture stated that the primary aim of the present enologists is to produce unique wines bearing the identical aroma characteristics of the particular type in question, and therefore one of the most important future tasks of yeast strain selection is to find and isolate the best Saccharomyces cerevisiae variants for the various varieties. 
The goal of our work was to find some yeast strains suitable for developing the characteristic fermentation products of certain Eger Region grape musts. In solving this problem a solution simpler than the yeast strain selection has been chosen. In 1996 and 1997 microvinification pilot fermentation experiments were performed by the lyophilized high quality yeast starter cultures of the firm Uvaferm in the Research Institute for Viticulture and Enology in Eger.

The firm wanted to explore which strains are the most suitable for the reductive fermentation of wines under the climatic and provincial conditions in question. Investigations have been carried out with the following grape varieties: Muscat Ottonel, Leányka and Chardonnay. The present paper reports on the Muscat Ottonel fermentation experiments in 1997.

For the fermentation of Muscat Ottonel must a strain producing elegant Muscat flavour and freshness had to be found. Secondary aroma substances synthesized by the yeast were not allowed to be too heavy and depress the identity of the wine.

The analysis used in the research is a special new sample preparation method, SPME, and the samples were measured with microbore column and EPC equipped GCFID instrument. Solid phase micro extraction (SPME) is a revolutionary new sample preparation procedure (YANG \& PEPPARD, 1994). The first wine aroma analytical application dealing with the SPME measurement of monoterpene-alcohol content can be found in the publication of DE LA CALLE GARCIA and co-workers (1996). Since that time head-space SPME has become a frequently applied preparatory method in wine analysis (VAs et al., 1998). It is very sensitive, fast, reproducible, non destructive and environmentally friendly procedure.

By using microbore (100 $\mu \mathrm{m}$ i.d.) capillaries, the time of analysis can be reduced by half or one third (appr. $15 \mathrm{~min}$ ) compared to the time required for conventional columns.

Fast sample preparation and analysis enabled us to measure the changes in aroma content of the microvinification experiments with satisfying frequency. Thus, the investigation has become suitable to follow the kinetics of the maturation process, too. Analysis has been aimed at examining the changes in aroma compounds of wines and allowing comparison of the final aroma content between different batches.

The change of individual primary aroma compounds (e.g. linalool, linalylacetate), that of the alcohols (fusel oils, iso-amylalcohol) ethyl- and acetate-esters synthesized during the fermentation have been determined.

Sensory evaluation of the wines has also been performed after the fermentation. 


\section{Materials and methods}

\subsection{Wines}

Wines (and previously grape musts) from the Muscat Ottonel grape variety were analysed in this study. Muscat Ottonel is a popular grape in the Eger wine region, Hungary. It exhibits a distinctive, strong muscat character. The grapes were picked on 16th September in 1997. Sugar content of grape was $190 \mathrm{~g}^{-1}$. The quality of grape was much better than in 1996, and this year we could start to control spontaneous fermentation also in our experiment. After the crushing of grape berries the juice was soaked for $4 \mathrm{~h}$ with the addition of $50 \mathrm{mg}^{-1} \mathrm{SO}_{2}$. The must was clarified overnight at cellar temperature. After the clarification process we prepared the following batches (we made two of each, and so the analytical results are the averages of two parallels):

- Spontaneous: Grape must fermented by natural yeast flora.

- Batch CM: Grape must fermented by Uvaferm CM yeast.

- Batch 228: Grape must fermented by Uvaferm 228 yeast.

- Batch ALB: Grape must fermented by Uvaferm ALB yeast.

The Uvaferm SC and CEG yeasts have not been used this year, because they yielded poor results with variety Muscat Ottonel in the previous year.

The yeast concentrations by inoculation of batches with starter cultures were regulated at $40 \mathrm{~g}^{-1}$ to eliminate the effect of natural flora.

The wines were fermented at $12{ }^{\circ} \mathrm{C}$ (cellar temperature).

\subsection{Sample preparation using SPME extraction}

Wine aroma analytes were concentrated with the SPME method according to the following protocol: each of the above mentioned sample categories were analyzed at nearly the same time. (This was possible due a very short chromatographic and sample preparation time). A volume of $2 \mathrm{ml}$ of each sample was placed in a $7 \mathrm{ml}$ sampling bottle. The SPME fiber was inserted into the head space and remained exposed there for $10 \mathrm{~min}$, while the liquid sample was subjected to low-speed agitation. The average temperature of the sample during this procedure was $20^{\circ} \mathrm{C}$.

Thermal desorption of analytes (when using SPME extraction) was performed in the chromatograph injector where the fiber remained for $5 \mathrm{~min}$ at $250^{\circ} \mathrm{C}$.

The fiber used was $100 \mu \mathrm{m}$ PDMS (polydimethylsixolane) phase coating (Supelco Inc., Bellefonte, PA, USA). It must be noted that PDMS coating is regularly used for the extraction of low molecular weight or volatile apolar compounds. 


\subsection{Instrumental analysis}

The gas-chromatograph was a Hewlett Packard (USA) 5890 series II Gas Chromatograph equipped with two channels EPC (Electronic Pressure Control). A Chrompack (Middleburg, The Netherlands) CP-WAX 52CB $10 \mathrm{~m} \times 0.1 \mathrm{~mm}$ fused capillary column coated with $0.2 \mu \mathrm{m}$ bonded polyethylene-glycol was used. The temperature of the injector was held constant at $250{ }^{\circ} \mathrm{C}$ during analysis. Splitless purge valve was closed for $5 \mathrm{~min}$. SPME splitless inlet liner was used with $0.75 \mathrm{~mm}$ internal diameter. The detector was a FID whose temperature was held constant at $280^{\circ} \mathrm{C}$.

$\mathrm{H}_{2}$ (purity 5.5) was used as the carrier gas: Gas linear velocity was $45 \mathrm{~cm} \mathrm{sec}^{-1}$ at 26.6 psig head-pressure. EPC had a controlled constant flow of $0.5 \mathrm{ml} \mathrm{min}^{-1}$.

The $\mathrm{GC}$ oven temperature was programmed as follows: initial: $35^{\circ} \mathrm{C}(0 \mathrm{~min}$ hold), 1 st ramp: $10^{\circ} \mathrm{C} \mathrm{min}^{-1}$ to $220^{\circ} \mathrm{C}(1 \mathrm{~min}$ hold $)$.

\subsection{Retention indices}

A test mixture containing 20 aliphatic hydrocarbons $\left(\mathrm{C}_{8}-\mathrm{C}_{27}\right)$ was used for external calibration, which was performed once a day. Retention indices were calculated with a software. Day to day reproducibility of retention indices determined in this way was \pm 2 unit (HARANGI \& VAS, 1998).

\section{Results and discussion}

The selected dried wine yeasts began the fermentation fast. The yeasts 228 and $\mathrm{CM}$ fermented with almost the same intensity. The slowest fermenting Uvaferm yeast was the ALB. The strain 228 showed the most reliable fermentation features. The natural yeast flora fermented in the most uncertain way (late start of fermentation possible oxidation problems; the fermentation was protracting).

The dominating monoterpene alcohols in the aroma profile of Muscat varieties are linalool, geraniol, nerol, citronellol and alpha-terpineol (RAPP, 1988). The changing in quantity of primary flavour materials and derivatives of them (linalool, nerol, geraniol, alfa-terpineol, citronellol) has been measured in the Muscat Ottonel samples under examination.

In Fig. 1 we can observe the changing of total quantity of terpene alcohols (linalool, terpineol, citronellol, geraniol). It is clearly observable that the quantity of terpenols in microvinification of wines is practically unchanged during the first $48 \mathrm{~h}$. After this time the concentration of terpene alcohols decreased greatly in the headspace of batches inoculated with three selected dried wine yeasts. The explanation of this is that the fermentation became faster and took place with higher intensity in the wines fermented by selected wine yeasts, and so the concentration of ethanol increased more 
quickly than in the spontaneous samples. Ethanol retains the terpene alcohols better in solution, according to the measuring results of DE LA CALLE GARCIA and co-workers, (1996) and so the concentration of the terpenols decreased only apparently in the steam space of wines fermented with Uvaferm yeasts. At the end of fermentation of all the samples, the wine with the highest total terpene content was produced by CM. The quantity of terpene alcohols was less in the wines produced by ALB, and even smaller concentrations were produced by 228 compared to $\mathrm{CM}$.

In spite of this, the alcohol content of the wine fermented by natural flora was the lowest (in this case the terpene alcohol concentration in the head space is greater at the same terpenol content), the quantity of measured terpenols and terpene derivatives was here found to be the lowest.

The analytical results supported the results of sensory evaluation of wines, because after the end of fermentation the most varietal character was shown by CM.

Ethyl-esters of straight-chain fatty acids and acetates of higher alcohols are the dominating esters of wines, and they are formed during the alcoholic fermentation (RAPP, 1988).

The fatty acid esters with the large number of combinations form the biggest group of aroma components between the secondary flavour materials. Examination of the changing quantities of ethyl-acetate, ethyl-butanoate, ethyl-hexanoate, -octanoate, -decanoate and diethyl-succinate was conducted in our microvinification experiments.

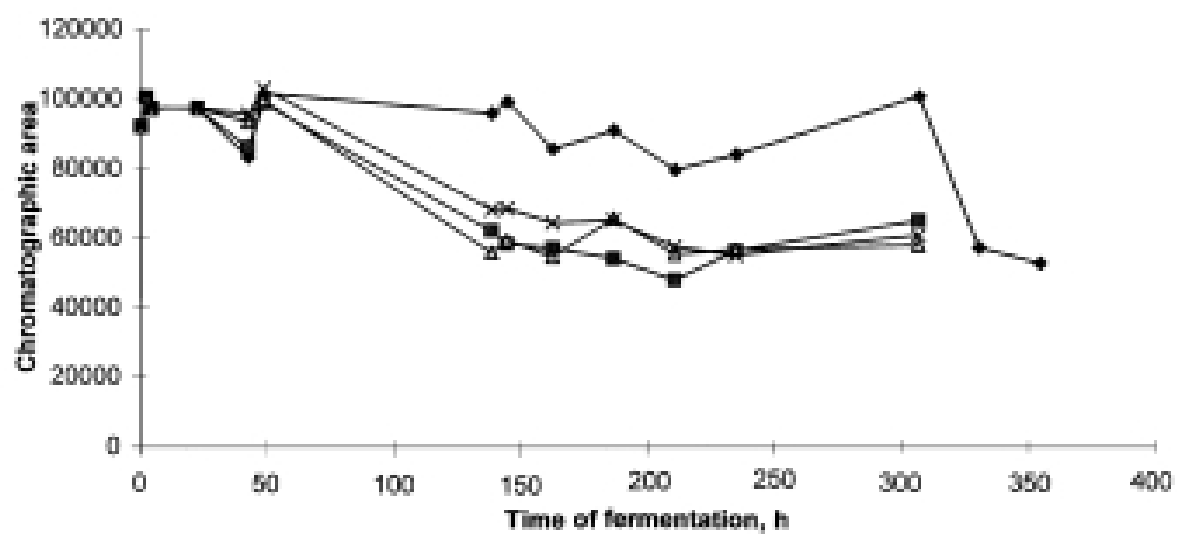

Fig. 1. Changing of terpene alcohols during the fermentation. $\bullet$ Control; $\mathbf{\square}$ : CM; $\Delta$ : 228; X: ALB. 


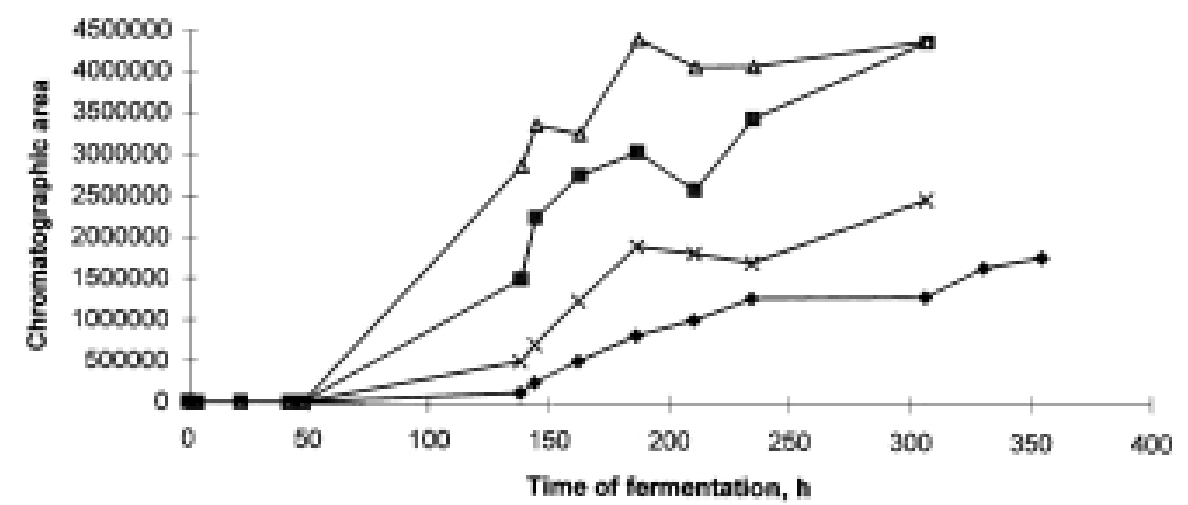

Fig. 2. Changing of ethyl-esters during the fermentation. $\bullet$ : Control; $\mathbf{\square}$ CM; $\Delta$ : 228; X: ALB.

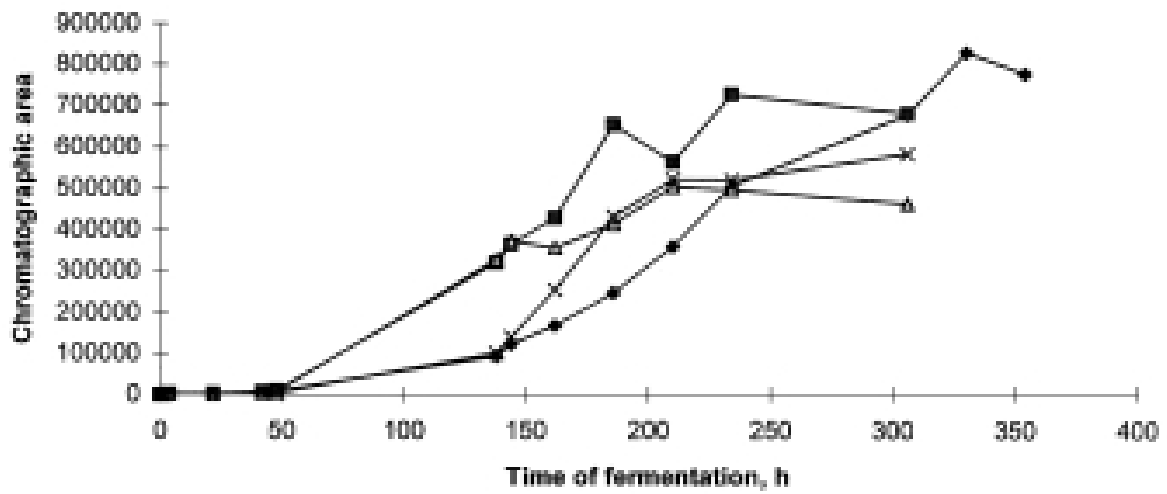

Fig. 3. Changing of acetate-esters during the fermentation. $\bullet$ : Control; $\mathbf{\square}$ : CM; $\Delta$ : 228; X: ALB.

Figure 2 displays changes in the total quantity of ethyl-esters during fermentation. These esters do not originate from the prefermentory process. The quantification of esters was measurable from the 48th hour. The strain 228 produced these compounds with the largest intensity. Not until the end of the fermentation did the CM reach the ethyl-ester concentration of the strain 228. The ALB produced less from these aroma components. The production of these aroma compounds of the control was the smallest.

The quantity of the following acetate-esters was determined in our measurements: linalil-acetate, hexyl-acetate, isoamyl-acetate, buthyl-acetate. Figure 3 shows the quantitative changes of acetate-esters with time. Though the total intensity of 
these compounds is only $1 / 5$ th of the intensity of ethyl-esters, they are important flavour materials. The acetate-esters give the fruity character to the wines.

The concentration of acetate-esters increased most dramatically with yeasts 228 and $\mathrm{CM}$ during the first $100 \mathrm{~h}$ of the fermentation. After this the intensity of acetateester production of strain 228 went back, and at the end of the fermentation the concentration of these materials was the lowest for this type of yeast. Not until the end of fermentation did the ALB produce more fruity esters than the 228. The CM and the sample with spontaneous fermentation was the richest in acetates.

Figure 4 summarizes the quantity formation of the aroma materials.

Besides the above mentioned aroma compounds the hexanol, octanol (primary aroma materials), fusel alcohols, phenylethyl-alcohol etc. were measured and there were numerous aroma compounds which we could not identify, because we did not have standards for these combinations.

The ethyl- and acetate-esters gave $65-70 \%$ of the total aroma quantity in our measurements. In compliance with it, the total aroma content in the samples changed with almost the same dynamics, like the whole ester content.

The aroma content of the batches were practically unchangeable until the 48th hour. After this the two fastest fermenting strains were the CM and 228. The intensity of aroma production of $\mathrm{CM}$ and 228 yeasts were the largest. At the end of fermentation, the wines produced by CM and 228 contained the most aroma. The ALB, compared with the most aromatic sample, produced $20-25 \%$ less flavour materials. The spontaneous fermentation gave 30-35\% less aroma than the CM and 228.

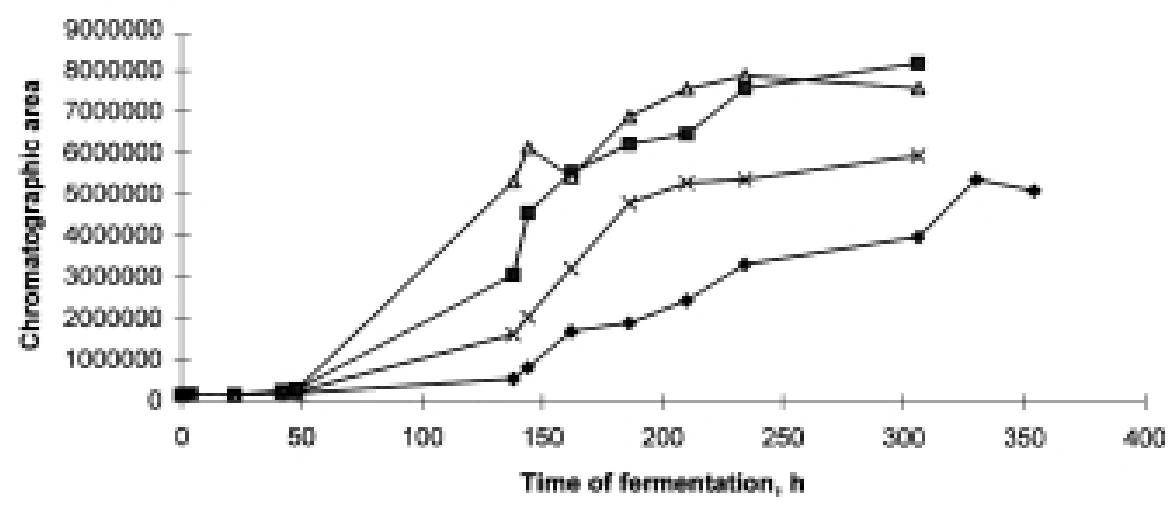


The result of sensory evaluation of wines was similar to our findings. The most aromatic wines were the batches fermented by $\mathrm{CM}$. The only problem with this yeast was that the wines of it were very poor in extract, and it can be a problem for the qualification of wine, because, according to the Hungarian regulations the wines fermented by $\mathrm{CM}$ can only be table wines, not quality wines. The second most aromatical wines were produced by the yeast 228 . This strain brought enough extract to the wines, and so the 228 was the most suitable yeast for the fermentation of Muscat Ottonel from the 5 tested strains during the two vintages. The spontaneous flora produced organoleptically more aromatical wines than the ALB, against the analytically measured results.

Special thanks to Chrompack company for a $100 \mu$ m i.d. CP-WAX 52CB capillary column.

\section{References}

DE la CAlle GarCia, D., Magnaghi, S., REICHENBäCHER, M. \& DANZER, K. (1996): Systematic optimization of the analysis of wine bouquet components by solid-phase microextraction. J. High. Resol. Chromatogr., 19, 257-262.

DITTRICH, H. H. (1992): Gärungsprodukt-Bildung und Aroma-Beeinflussung durch Reinzucht-Hefen. Symposium Lallemand: Sensory contribution of yeast to wine, Weissenkirchen/Wachau (Austria) May 21-24, 1992.

HARANGI, J. \& VAS, GY. (1998): Identification of GC peaks - identification of GC chromatogram. 20th International Symposium on Capillary Chromatography, poster No. A11.

RAPP, A. (1988): Wine aroma substances from gas chromatographic analyses. -in: LISKENS, H. F., JACKSON, J. F. (Eds) Wine analyses. Springer Verlag Berlin, Heidelberg, pp. 29-66.

YANG, X. \& PEPPARD, T. (1994): Solid phase microextraction for flavor analysis. J. agric. Fd. Chem., 42, 1925-1930.

VAS, GY., KÖTELEKY, K., FARKAS, M., DOBÓ, A. \& VÉKEY, K. (1998): Fast screening method for wine headspace compounds using solid-phase microextraction (SPME) and capillary GC technique. Am. J. Enol. Vitic., 49, 100-104. 